\title{
Structure of ice confined in carbon and silica nanopores
}

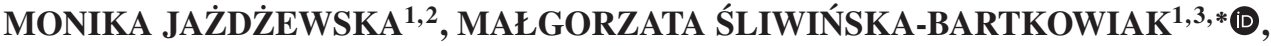 \\ KAMILA DOMIN ${ }^{1,3}$, DOROTA M CHUDOBA ${ }^{1,2}$, ANATOLY I BESKROVNYI ${ }^{2}$, DIMITR S NEOV ${ }^{2,4}$ \\ and KEITH E GUBBINS ${ }^{5}$ \\ ${ }^{1}$ Faculty of Physics, Adam Mickiewicz University, 61-614 Poznan, Poland \\ ${ }^{2}$ Frank Laboratory of Neutron Physics, Joint Institute for Nuclear Research, Dubna 141980, Russia \\ ${ }^{3}$ NanoBioMedical Centre, Adam Mickiewicz University, 61-614 Poznan, Poland \\ ${ }^{4}$ Institute for Nuclear Research and Nuclear Energy, Sofia BG-1784, Bulgaria \\ ${ }^{5}$ Department of Chemical and Biomolecular Engineering, NC State University, Raleigh, NC 27695-7905, USA \\ *Author for correspondence (msb@amu.edu.pl)
}

MS received 27 September 2018; accepted 8 January 2019; published online 3 June 2019

\begin{abstract}
In this work, water confined in silica and carbon nanopores has been examined. The purpose of this study is to describe the melting behaviour and structure of ice confined in silica nanopores, KIT-6 and ordered carbon nanopores, CMK-3, having pore diameters of 5.9 and $5.2 \mathrm{~nm}$, respectively. To determine the melting temperature of ice inside the nanopores, we performed differential scanning calorimetry measurements of the systems studied. We found that the melting temperature of confined ice is reduced relative to the bulk melting point and this shift is $16 \mathrm{~K}$ for water confined in KIT- 6 and $21 \mathrm{~K}$ for water confined in CMK-3. The structural properties of water at the interfaces were analysed by using the neutron diffraction method (ND). The ND measurements for all the systems studied, showed the features of both hexagonal ice, $I_{\mathrm{h}}$, and cubic ice, $I_{\mathrm{c}}$. However, we show that the ice confined in nanopores does not have a structure corresponding to the typical hexagonal form or the metastable cubic form. The ice confined in nanopores has a structure made up of cubic sequences interlaced with hexagonal sequences, which produce the stacking disordered ice (ice $I_{\mathrm{sd}}$ ).
\end{abstract}

Keywords. Nanophase; ice structure; carbon and silica nanopores; neutron diffraction; effect of confinement; cubicity.

\section{Introduction}

The phase diagram of ice shows that they have many different crystal forms; these forms depend on the local molecular correlations that influence the ordered arrangement of hydrogen-bonded configurations. There are 15 crystalline phases of ice [1], 3 polyamorphic states of ice [2] and high viscosity liquid phases [3] that are known. A lot of these structures are high-pressure and low temperature forms of ice.

In general, it is assumed that under ambient conditions, ice exists in two crystalline forms: stable hexagonal ice (ice $I_{\mathrm{h}}$ ) and metastable cubic ice (ice $I_{\mathrm{c}}$ ). Usually, water freezes to hexagonal ice which crystallizes in the space group $P 63 / \mathrm{mmc}$, but under certain conditions, mostly in laboratory, it can freeze to create cubic ice in the space group $F d 3 m$ [4]. Both hexagonal and cubic ice consist of layers composed of six-membered rings of hydrogen-bonded water molecules; however, they differ in the arrangement of second-neighbours layer [5].

There are several ways by which ice $I_{\mathrm{c}}[6]$ can be formed [7]. It can be obtained, for example, from vapour deposition [6] and in addition by the warming of amorphous ices $[8,9]$ and recovered high-pressure forms of ice $[10,11]$ and by freezing of nanometre-scale water clusters $[12,13]$. Recently, the creation of a cubic form of ice by freezing of water confined in nanopores has also been also reported [14-16]. Cubic ice is usually obtained in the form of very small crystallites that lead to particle size broadening of the diffraction pattern [17]. It is considered that sample size (i.e., droplet or pore size) plays an important role in the formation of ice $I_{\mathrm{c}}$. It was found that transitions starting from a solid water phase which lead to ice $I_{\mathrm{c}}$ are irreversible [7].

Cubic structure or a defective form of cubic ice is usually formed in nanometre-sized confined geometry $[15,16,18$ 24]. Seyed-Yazdi et al $[25,26]$ showed that the nucleation of water confined in silica nanopores creates a mixture that incorporates hybrid crystallites of ice $I_{\mathrm{h}}$ and ice $I_{\mathrm{c}}$ with an intermediate density form of amorphous ice. Liu et al [18] claimed that in the case of the over-filled sample, the water inside pores freezes to form a defective cubic form of ice, while the formation of a hexagonal structure is observed outside of the silica grains. While using molecular dynamics simulations, Moore et al [27] found that the ratio of cubic to hexagonal layers in confined ice is about 2:1. Moreover, the structure of ice confined in hydrophilic nanopores of $3 \mathrm{~nm}$ diameter has stacking faults.

It is considered that the formation of cubic structures, rather than hexagonal structures, in the pores can be attributed to the restrictions on the size of the crystallite. However, according 
to recently published studies $[4,10,28-31]$, the cubic form that has been observed is not exactly cubic. This form of ice, so-called cubic phase, shows stacking faults instead of an ordered arrangement of two-dimensional layers [28]. It is clear that the form of ice which was called cubic ice in the past is, in fact, a stacking-disordered material containing cubic sequences interlaced with hexagonal sequences [31]. Kuhs et al [10] noticed that their samples of ice $I$, which they referred to as ice $I_{\mathrm{c}}$, contained some deformation faults in the $I_{\mathrm{c}}$ form. These stacking faults are the result of randomly dispersed sequences of hexagonal ice in the structure of cubic ice. Hansen et al [17,32] quantitatively showed that all the noncubic features in ice $I$, formed from ice V and ice IX, could be the result of the stacking disorder and the so-called cubic ice is not cubic, but trigonal (space group $P 3 m 1$ ). In previous literature on the subject, we can find two main nomenclatures that have been suggested for stacking disordered ice $I$. Malkin et al [31] proposed the abbreviation ice $I_{\text {sd }}$. This term includes both ice $I_{\mathrm{c}}$ with varying amounts of ice $I_{\mathrm{h}}$ sequences and ice $I_{\mathrm{h}}$ containing varying amounts of ice $I_{\mathrm{c}}$ sequences. Hansen et al [33] suggested another term that may be seen as a continuum from ideal ice $I_{\mathrm{c}}$ to ideal ice $I_{\mathrm{h}}$. They analysed the effect of different stacking disorder interactions on the diffraction patterns of stacking disordered ice and proposed to name it ice $I_{\mathrm{ch}}$. The propensity of ice $I$ to grow with stacking disorders has been observed in a large number of experimental and simulation studies $[5,7,15,27,34-41]$.

It is known that water confined in a nanoscale environment exhibits unique properties and has been the subject of much attention. The behaviour of the nanoconfined phase is different from the behaviour of the bulk phase, which occurs due to the size effect and competition between the fluid-wall and fluid-fluid intermolecular interactions. In the phase transition phenomena it can lead to the decreasing of the melting temperature of adsorbate relative to the bulk melting point [42-47].

\section{Methods}

We report on the experimental studies of the melting transition and structure of ice confined in silica and cylindrical carbon nanopores of similar inner diameter: 5.9 and $5.2 \mathrm{~nm}$, respectively. The experimental methods used for the studies are: differential scanning calorimetry (DSC) and neutron diffraction (ND).

\subsection{Materials}

In our experiments, we have used silica nanopores of type KIT-6 and mesoporous carbon material CMK-3, which are the reverse carbon replica of silica mesopores, SBA-15. The silica mesopores SBA-15 and KIT-6 were synthesized according to the literature procedure $[48,49]$. As a carbon precursor, in the case of CMK-3 nanopores, furfuryl alcohol was used [50]. The materials were characterized by several methods

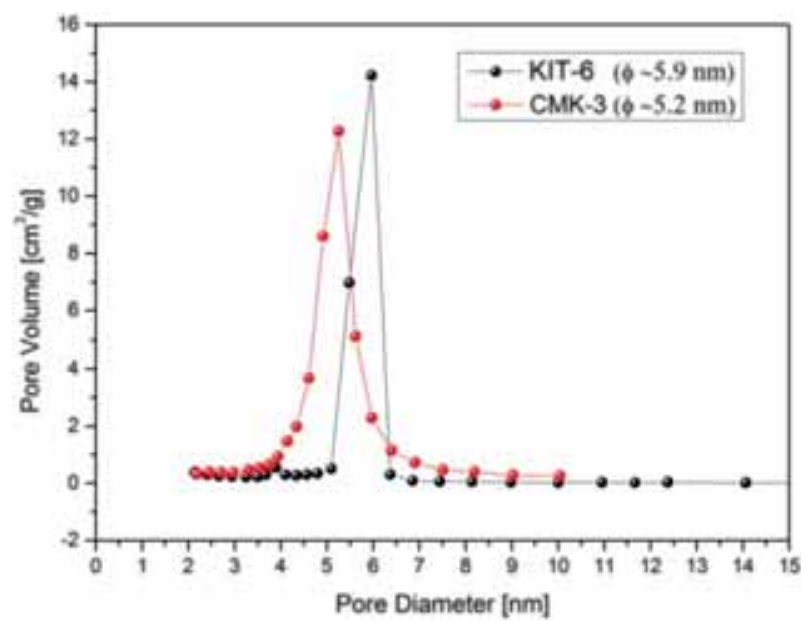

Figure 1. Pore size distribution calculated for the CMK-3 and KIT6 matrices.

e.g., transmission electron microscopy and small angle $\mathrm{X}$-ray scattering and nitrogen sorption analysis (Micrometrics ASAP 2020). The specific surface area was calculated using the Brunauer-Emmet-Teller (BET) method and pore volume and pore size distribution were derived using the Barrett-Joyner-Halenda (BJH) method [50]. The pore size distributions calculated for the samples of CMK-3 $(5.2 \mathrm{~nm})$ and KIT-6 $(5.9 \mathrm{~nm})$ are presented in figure 1 .

Porous samples were heated to about $400 \mathrm{~K}$ and kept under vacuum $\left(10^{-3}\right.$ torr) for several days to remove air prior to and during the introduction of $\mathrm{D}_{2} \mathrm{O}$ (100\% deuterium purchased from Sigma) or deionized $\mathrm{H}_{2} \mathrm{O}$ water. The samples for ND measurements were then hydrated in a humid atmosphere at room temperature for a few days. The hydrated process was continued until the sample mass increased by about $70 \%$. This way of sample preparation allowed us to obtain samples with $\mathrm{D}_{2} \mathrm{O}$ only inside the pores, and there was no excess water outside the pores. For DSC measurements, the carbon materials were filled with deionized water under vacuum by using a special tube. This method of filling the carbon nanopores with water ensured very good pore saturation. As a result, we obtained samples that contained water inside and outside the pores which allowed us to determine the shift of the melting temperature of water in the pores and directly compare it to the bulk melting point.

\subsection{DSC measurements}

The analysed systems were characterized by using DSC and ND methods. The melting behaviour of the confined water was studied by the DSC method. A PerkinElmer DSC 800 advanced double-furnace DSC was used to determine the melting temperature of ice confined in nanopores. The measurements were performed at temperature ranging between 173 and $313 \mathrm{~K}$ with a heating rate of $10 \mathrm{~K} \mathrm{~min}^{-1}$. The 
melting temperatures were determined from the position of the peaks of the heat flow signals on a heating thermogram.

\subsection{ND method}

The crystal structure of confined ice was determined by the ND method. $\mathrm{D}_{2} \mathrm{O}$ water was used in the neutron scattering study to avoid the complications of incoherent scattering and inelasticity corrections. The ND measurements were performed in the real-time powder diffractometer $[51,52]$ at the Frank Laboratory of Neutron Physics of the Joint Institute for Nuclear Research (JINR) with the fast-pulsed reactor IBR-2. The resolution of the diffraction patterns was determined by the width of the pulse from the neutron source, $\Delta d / d=0.01$, in the range of interplanar spacings, $d_{h k l}$, from 1 to $20 \AA$. The scattering angle, $2 \theta=170^{\circ}$ was fixed. The sample was placed in a vanadium container with a diameter of $3 \mathrm{~mm}$ and a height of $25 \mathrm{~mm}$ and was cooled to about $100 \mathrm{~K}$ and then warmed to $300 \mathrm{~K}$. Structure refinement was carried out using the FullProf program based on the multi-phase Rietveld analysis method $[53,54]$.

\section{Results and discussion}

The melting behaviour of water confined in KIT- 6 and CMK-3 nanopores was determined using the DSC method. The DSC scans corresponding to the melting of $\mathrm{H}_{2} \mathrm{O}$ in silica and carbon pores are presented in figure 2 .

The endothermic peaks which occur at the temperature of $257 \mathrm{~K}$ in the case of KIT- 6 and at $252 \mathrm{~K}$ for CMK-3 are related to the melting temperature of the confined ice. We also observed an endothermic peak at a temperature of $273 \mathrm{~K}$ which is connected with the bulk melting point of ice. The shift of the melting temperatures towards lower temperatures for $\mathrm{H}_{2} \mathrm{O}$ in KIT-6 and CMK-3 mesopores relative to bulk water is -16 and $-21 \mathrm{~K}$, respectively ( $\Delta T=T_{\text {mbulk }}-T_{\text {mpores }}$ ).

To determine the structure of confined ice, the ND method was used which provided information on the long-range order in crystal structures. The diffraction spectra indicate whether the studied materials satisfied the Bragg's law. While studying an ordered crystal material, sharp diffraction peaks are observed. When the long-range order in a crystal breaks, it influences the diffraction pattern.

In figure 3, the diffraction patterns for $\mathrm{D}_{2} \mathrm{O}$ in KIT-6 and CMK-3 at a temperature of $270 \mathrm{~K}$ are presented. At $270 \mathrm{~K}$, we did not observe crystalline peaks (the ice in the pores is in liquid phase), so the diffraction pattern is typical for the amorphous liquid state.

The ND patterns for $\mathrm{D}_{2} \mathrm{O}$ water in KIT- 6 and CMK-3 at $100 \mathrm{~K}$ is presented in figure 4.

Figure 4 shows the crystalline forms of water. The main triplet shows the features of the hexagonal ice with space group $P 63 / \mathrm{mmc}$ and peaks at the positions: $d=3.90,3.66$ and $3.44 \AA$ corresponding to the main reflections of planes [100], [002] and [101] for both the studied systems.
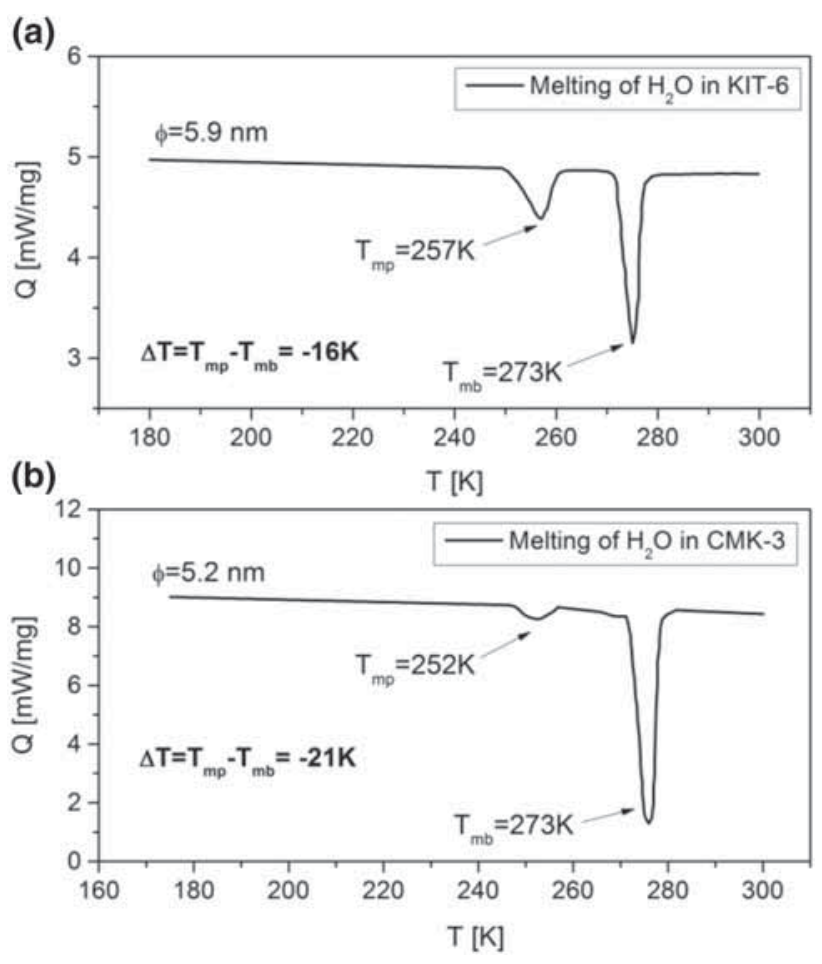

Figure 2. DSC scan for $\mathrm{H}_{2} \mathrm{O}$ confined in (a) KIT-6 and (b) CMK-3 with mesopores of diameter of 5.9 and $5.2 \mathrm{~nm}$, respectively.

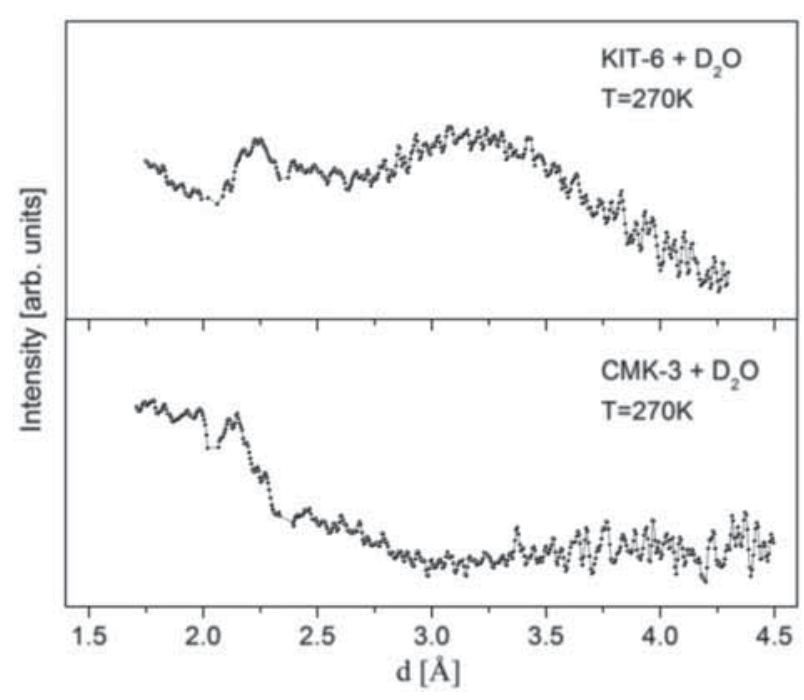

Figure 3. ND results for $\mathrm{D}_{2} \mathrm{O}$ in KIT-6 and CMK-3, measured at $270 \mathrm{~K}$.

However, the widths of the observed peaks are broader relatively to the hexagonal crystal structure. In addition, the intensity of the experimental peaks does not correspond to the diffraction pattern for hexagonal ice [18] and does not match the calculated patterns that are shown in figure 5. In table 1, the structural parameters of the hexagonal $I_{\mathrm{h}}$ and cubic $I_{\mathrm{c}}$ phases are presented. 
(a)

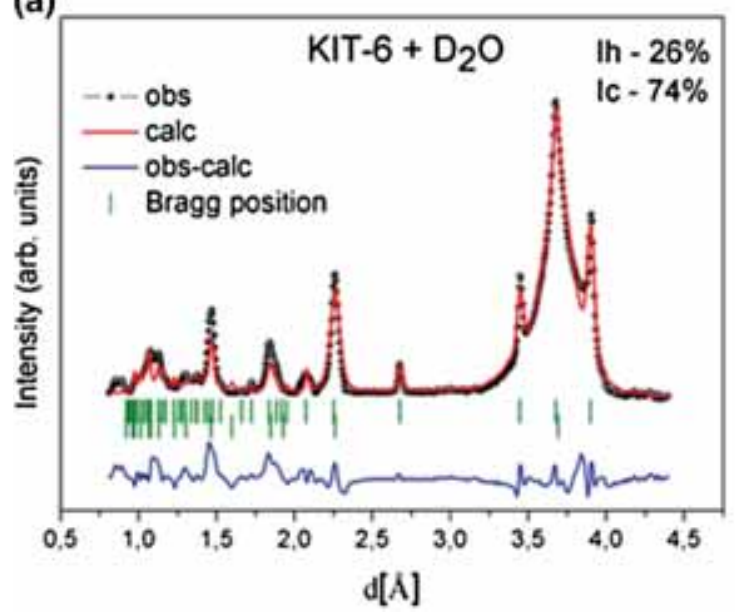

(b)

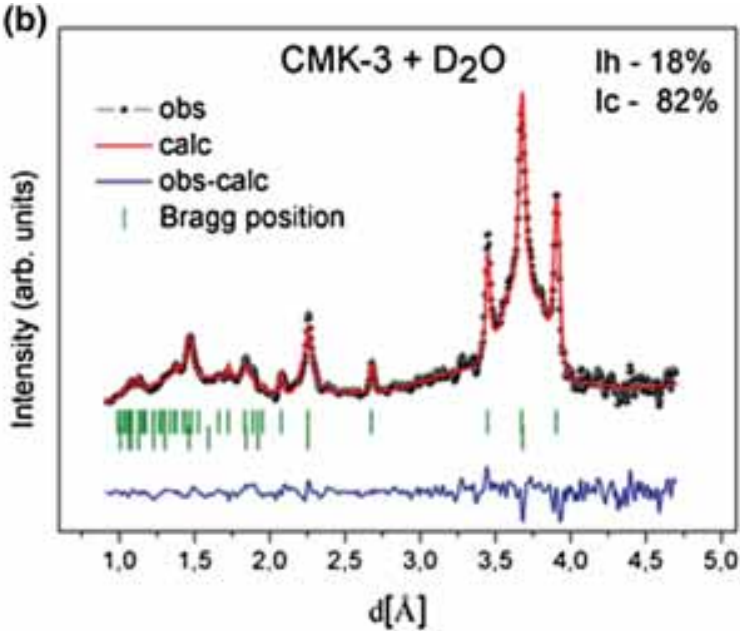

Figure 4. ND patterns and the results of Rietveld refinement of $\mathrm{D}_{2} \mathrm{O}$ in (a) KIT-6 and (b) CMK-3 at $T=100 \mathrm{~K}$ and the percentage composition of ice $I$ phases (the blue dotted line shows the difference between the experimental spectrum and Rietveld refinement).

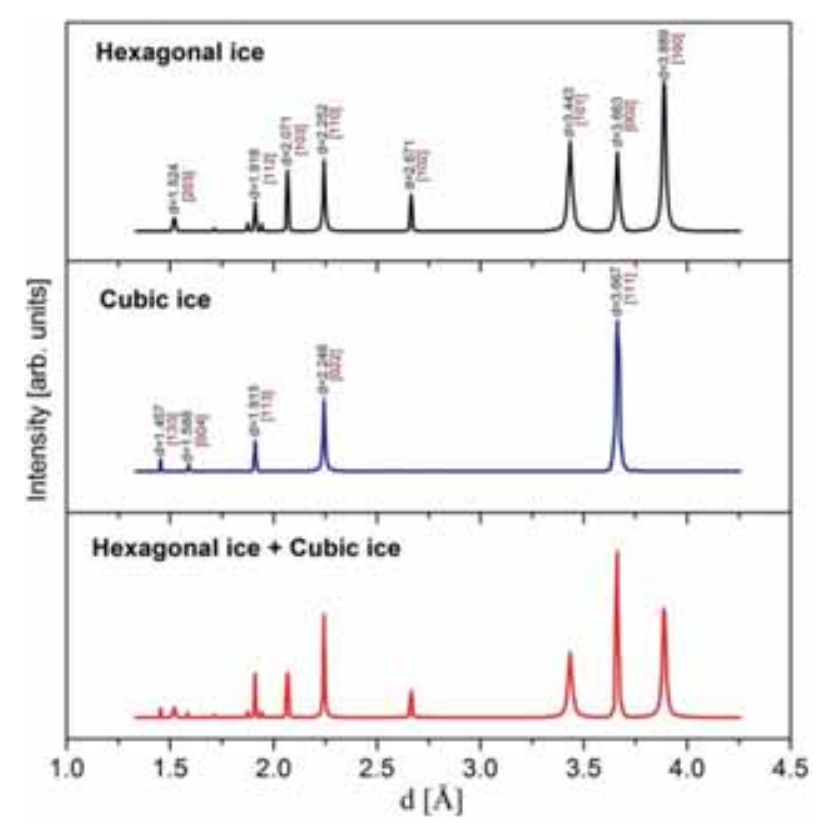

Figure 5. The calculated patterns for (a) hexagonal ice structure, (b) cubic ice structure and (c) superposition of $I_{\mathrm{h}}$ and $I_{\mathrm{c}}$.

In figure 5a, the diffraction pattern calculated on the basis of the Mincryst Crystallographic Database for hexagonal ice is presented.

In figure $5 b$, the calculated diffraction pattern for cubic ice with the space group $F d 3 m$ is presented and in figure 5c, the superposition of these two forms of ice is shown. In the experimental diffraction patterns shown in figure 4 , the peaks in the centre of the triplet $(d=3.66 \AA)$ can be a superposition of the cubic and hexagonal ice structures, comparable with the diffraction pattern shown in figure $5 \mathrm{c}$. The peaks in further positions ( $d=2.24$ and $1.83 \AA$ ), which are typical of cubic ice as well as of hexagonal ice structures, also showed a significant broadening when compared with the calculated diffraction patterns, which is suggestive of some disorder in the crystal. As we can see from the experimental profiles (figure 4), the peaks that can be treated as a superposition of the cubic and hexagonal forms of ice $(d=3.66,2.24$ and $1.83 \AA)$ are significantly broadened and indicative of a defective structure of ice, which cannot be a simple mixture of cubic and hexagonal ice structures. Such a defective structure of the cubic ice was also observed in previous experiments on confined ice formation $[15,18,25]$. Recently published results of X-ray diffraction studies for water confined in nanopores $[55,56]$ and the theoretical studies by Malkin et al $[31,38]$ suggest that the obtained profile can be a hybrid of both hexagonal and cubic ice forms that were formed as a result of stacking faults. The theory [38] predicts that when a water droplet freezes, a two-dimensional nucleation process occurs. As a result, the layers of hexagonal and cubic ice structures grow alternately in the crystal (in various combinations) and the resulting ice is made up of a combination of intertwined cubic and hexagonal stacking sequences. The name proposed for this complex structure of ice is disordered stacking ice, $I_{\text {sd }}$. It is precisely these faults that lead to the formation of a defective lattice structure.

The FullProf program was used to fit both the intensities and peak positions of the investigated samples. We used this program to assess the contribution of hexagonal ice and cubic ice in $I_{\mathrm{sd}}$ for the analysis of the diffraction pattern obtained. We have noticed that the ice confined in pores of KIT-6 contains about $26 \%$ hexagonal ice and $74 \%$ cubic ice fractions, whereas in the case of ice confined in the pores of CMK-3, we have observed about $18 \%$ hexagonal ice and $82 \%$ cubic $I_{\mathrm{c}}$ fractions. The Bragg $R$ factors from the analysis of data were $7.6\left(I_{\mathrm{h}}\right)$ and 10.8 
Table 1. Structural parameters of hexagonal $I_{\mathrm{h}}$ and cubic $I_{\mathrm{c}}$ phases.

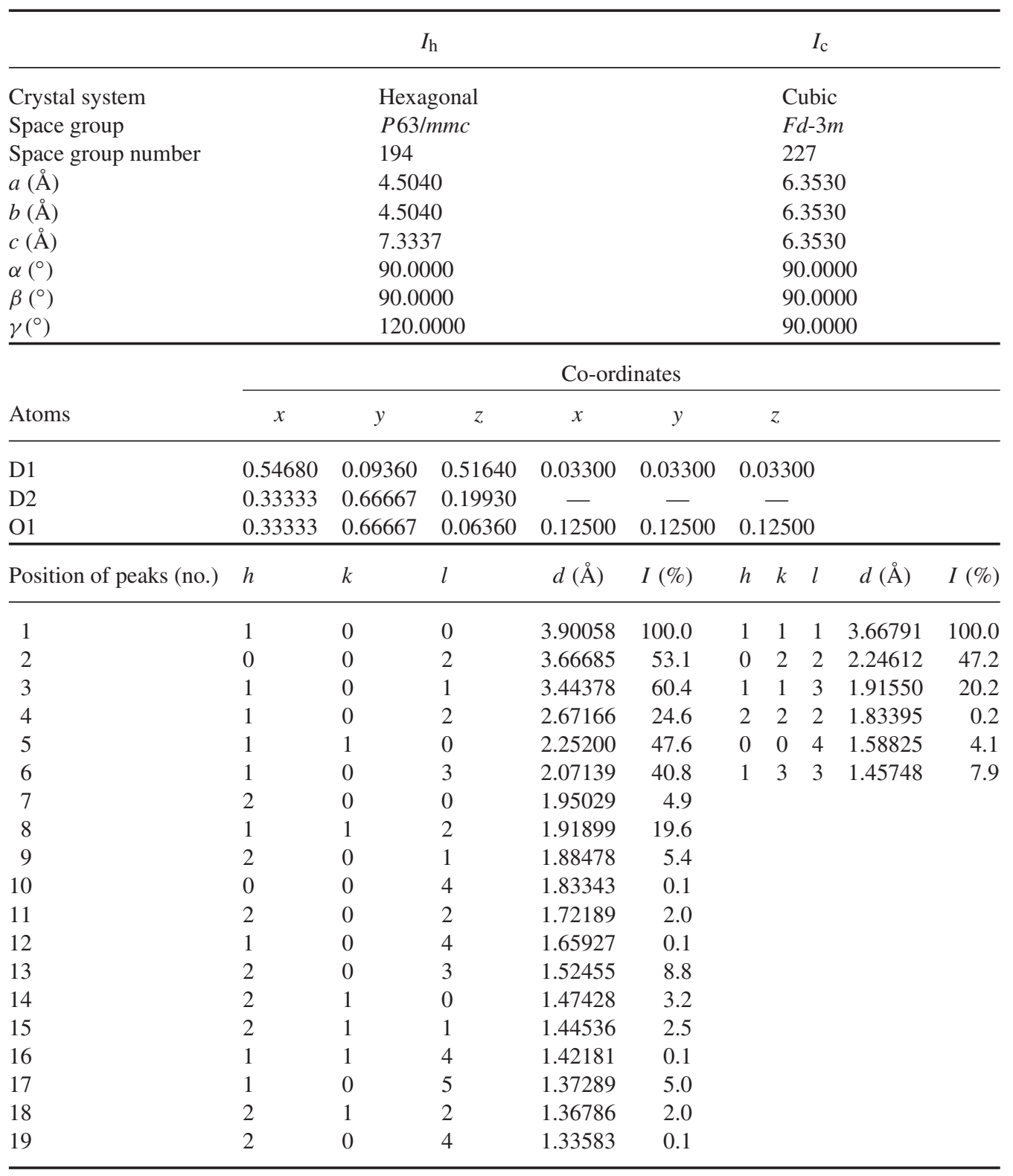

$\left(I_{\mathrm{c}}\right)$ for CMK-3 and $7.9\left(I_{\mathrm{h}}\right)$ and $16.9\left(I_{\mathrm{c}}\right)$ in the case of KIT-6. In figure 4, the Rietveld refinement for $\mathrm{D}_{2} \mathrm{O}$ in KIT-6 (figure 4a) and CMK-3 (figure $4 \mathrm{~b}$ ) at $T=100 \mathrm{~K}$ and the percentage composition of the phases (cubic and hexagonal ice) are presented.

As can be seen from figure 4 , for $\mathrm{D}_{2} \mathrm{O}$ in KIT-6, we observed the presence of $74 \%$ of the cubic phase and $26 \%$ of the hexagonal phase ice fractions and for $\mathrm{D}_{2} \mathrm{O}$ in CMK-3, we observed $82 \%$ of the cubic phase and $18 \%$ of the hexagonal phase fractions. Our results showed that the cubicity depends on the kind of pore walls as the pore sizes are similar for both porous matrices. We can see that the silica pores are more preferential for the hexagonal ice formation than the carbon walls of the pores. The relationship between the cubicity of ice $I$ and the corresponding degree of stacking disorder should be taken into consideration when analysing the structure of ice. According to Carr et al [5], the highest stacking disorder state is reached at cubicity of $50 \%$ in ice. When the ratio of cubic ice increases relative to hexagonal ice, the stacking disorder decreases until the perfect $I_{\mathrm{c}}$ structure is attained, when $I_{\mathrm{c}}$ is free of any stacking disorders. Also, in pure ice $I_{\mathrm{h}}$, when the cubicity is zero, there is no stacking disorder present. It was found that the small size of drops can play a role in increasing cubicity. Ice confined in nanopores becomes more cubic as the pore size decreases $[14,15]$. A similar dependence was shown by Domin et al [55]; when water is confined in smaller pores, the calculated percentage composition of the cubic form of ice increases. Malkin et al [38] showed that 
the ice produced from homogeneous nucleation in pure water droplets at around $230 \mathrm{~K}$ was fully random, with $50 \%$ cubic and $50 \%$ hexagonal stackins. Hansen et al [32] were able to show the probabilities of occurrence of cubic and hexagonal sequences in ice formed from ice $\mathrm{V}$ and ice IX. These probabilities show that cubic sequences are present up to $\sim 60 \%$ in ice $I_{\mathrm{sd}}$ formed from ice $\mathrm{V}$. They also indicated that the correlations between stacking faults are different depending on the route of formation. Furthermore, Moore et al [27] showed that the number of cubic layers of ice in the pores is much higher that the number of hexagonal layers. On the basis of our study, we were able to show that in the confined space, the type of pore surfaces (silica and carbon) can control the ratio of the cubic and hexagonal ice in $I_{\mathrm{sd}}$ ice, even in the case where pore sizes are very similar.

\section{Conclusion}

Our DSC results indicated a reduction in the melting temperature of water confined in the ordered silica and carbon mesopores. This effect can be explained in terms of the fluidwall attractive interaction and agrees with previous results [42-47]. ND research, which was made at temperatures below the pore melting temperature, provides a strong evidence of the presence of both hexagonal and cubic ice inside the pores. We were not able to obtain a good Rietveld refinement of our diffraction patterns with typical hexagonal and cubic separated ice forms. Our diffraction data show the existence of features (such as diffraction profile broadening) of stacking disorder ice $I_{\text {sd }}$ confined in pores. The asymmetric profile that has been associated with the presence of some defects or disorders in the confined ice structure was also observed in previous studies which concern the ice in nanopores [23,25,26,55]. We evaluated the percentage contribution of cubic and hexagonal ice inside silica and carbon mesopores. The results showed a higher contribution of the cubic form in the case of carbon pores compared with the silica pores, which is related to the degree of disordering of the crystal structure.

Knowledge of the process of formation of stacking faults, which are a type of disorder at a nanometre scale in crystals, is crucial for the production of technologically important materials, whose properties significantly depend on such a disorder.

\section{Acknowledgements}

Financial support from the National Center of Science under grants DEC-2013/09/B/ST4/03711 and UMO-2016/22/A/ ST4/00092 and from the grant of Research Group at JINR and Research Centers in Poland under grant number 04-4$1121-2015 / 2020$ is gratefully acknowledged. This work was also partially supported by the International PhD Projects Programme of the Foundation for Polish Science operated within the Innovative Economy Operational Programme (IE
OP) 2007-2013 within the European Regional Development Fund.

\section{References}

[1] Salzmann C G, Radaelli P G, Slater B and Finney J L 2011 Phys. Chem. Chem. Phys. 1318468

[2] Loerting T, Winkel K, Seidl M, Bauer M, Mitterdorfer C, Handle P H et al 2011 Phys. Chem. Chem. Phys. 138783

[3] Palmer J C, Martelli F, Liu Y, Car R, Panagiotopoulos A Z and Debenedetti P G 2014 Nature 510385

[4] Johari G P and Andersson O 2015 J. Chem. Phys. 143054505

[5] Carr T H G, Shephard J J and Salzmann C G 2014 J. Phys. Chem. Lett. 52469

[6] König H 1943 Z. Kristallogr. 105279

[7] Kuhs W F, Sippel C, Falenty A and Hansen T C 2012 Proc. Natl. Acad. Sci. 10921259

[8] Dowell L G and Rinfret A P 1960 Nature 1881144

[9] Mcmillan J A and Los S C 1965 Nature 206806

[10] Kuhs W F, Bliss D V and Finney J L 1987 J. Phys. Colloq. 48 631

[11] Bertie J E, Calvert L D and Whalley E 1963 J. Chem. Phys. 38 840

[12] Huang J and Bartell LS 1995 J. Chem. Phys. 993924

[13] Bartell L S and Lennon P L 2009 J. Chem. Phys. 130084303

[14] Morishige K, Yasunaga H and Uematsu H 2009 J. Phys. Chem. C 1133056

[15] Morishige K and Uematsu H 2005 J. Chem. Phys. 12244711

[16] Steytler D C, Dore J C and Wright C J 1983 J. Phys. Chem. 87 2458

[17] Hansen T C, Koza M M and Kuhs W F 2008 J. Phys. Condens. Matter 20285104

[18] Liu E, Dore J C, Webber J B W, Khushalani D, Jahnert S, Findenegg G H et al 2006 J. Phys. Condens. Matter 1810009

[19] Dunn M, Dore J C and Chieux P 1988 J. Cryst. Growth 92233

[20] Dore J, Webber B, Hartl M, Behrens P and Hansen T 2002 Physica A $\mathbf{3 1 4} 501$

[21] Baker J M, Dore J C and Behrens P 1997 J. Chem. Phys. 101 6226

[22] Handa Y P, Zakrzewski M and Fairbridge C 1992 J. Phys. Chem. 968594

[23] Dore J 2000 Chem. Phys. 258327

[24] Takamuku T, Yamagami M, Wakita H, Masuda Y and Yamaguchi T 1997 J. Phys. Chem. B 1015730

[25] Seyed-Yazdi J, Farman H, Dore J C, Webber J B W and Findenegg G H 2008 J. Phys. Condens. Matter 20205108

[26] Seyed-Yazdi J, Farman H, Dore J C, Webber J B W, Findenegg G H and Hansen T 2008 J. Phys. Condens. Matter 20205107

[27] Moore E B, Llave E, Welke K, Scherlis D A and Molinero V 2010 Phys. Chem. Chem. Phys. 124124

[28] Fitzgerald R J 2013 Phys. Today 6616

[29] Amaya A J, Pathak H, Modak V P, Laksmono H, Loh N D, Sellberg J A et al 2017 J. Phys. Chem. Lett. 83216

[30] Lupi L, Hudait A, Peters B, Grünwald M, Mullen R G, Nguyen A H et al 2017 Nature 551218

[31] Malkin T L, Murray B J, Salzmann C G, Molinero V, Pickering S J and Whale T F 2015 Phys. Chem. Chem. Phys. 1760

[32] Hansen T C, Koza M M, Lindner P and Kuhs W F 2008 J. Phys. Condens. Matter 20285105 
[33] Hansen T C, Sippel C and Kuhs W F 2015 Z. Kristallogr. 230 75

[34] Hansen T C, Falenty A and Kuhs W F 2007 in Physics and chemistry of ice W F Kuhs (ed.) (Cambridge: Royal Society of Chemistry) p 201

[35] Kuhs W F, Genov G, Staykova D K and Hansen T 2004 Phys. Chem. Chem. Phys. 64917

[36] Kohl I, Mayer E and Hallbrucker A 2000 Phys. Chem. Chem. Phys. 21579

[37] Hudait A, Qiu S, Lupi L and Molinero V 2016 Phys. Chem. Chem. Phys. 189544

[38] Malkin T L, Murray B J, Brukhno A V, Anwar J and Salzmann C G 2012 Proc. Natl. Acad. Sci. 1091041

[39] Moore E B and Molinero V 2011 Phys. Chem. Chem. Phys. 13 20008

[40] González Solveyra E, Llave E, Scherlis D A and Molinero V 2011 J. Phys. Chem. B 11514196

[41] Johnston J C and Molinero V 2012 J. Am. Chem. Soc. 134 6650

[42] Alba-Simionesco C, Coasne B, Dosseh G, Dudziak G, Gubbins K E, Radhakrishnan R et al 2006 J. Phys. Condens. Matter 18 R15

[43] Gelb L D, Gubbins K E, Radhakrishnan R and SliwinskaBartkowiak M 1999 Rep. Prog. Phys. 621573
[44] Radhakrishnan R, Gubbins K E and Sliwinska-Bartkowiak M 2002 J. Chem. Phys. 1161147

[45] Sliwinska-Bartkowiak M, Dudziak G, Sikorski R, Gras R, Radhakrishnan R and Gubbins K E 2001 J. Chem. Phys. 114950

[46] Morishige K, Yasunaga H and Matsutani Y 2010 J. Phys. Chem. C 1144028

[47] Sliwinska-Bartkowiak M, Jazdzewska M, Huang L L and Gubbins K E 2008 Phys. Chem. Chem. Phys. 104909

[48] Jun S, Joo S H, Ryoo R, Kruk M, Jaroniec M, Liu Z et al 2000 J. Am. Chem. Soc. 12210712

[49] Kleitz F, Choi S H and Ryoo R 2003 Chem. Commun. 172136

[50] Domin K $2016 \mathrm{PhD}$ thesis (A. Mickiewicz University)

[51] Balagurov A M, Beskrovnyy A I, Zhuravlev V V, Mironova G M, Bobrikov I A, Neov D et al 2016 J. Surf. Invest.: X-ray, Synchrotron Neutron Tech. 10467

[52] Real-Time Neutron Diffractometer. Available: http://flnph.jinr. ru/en/facilities/ibr-2/instruments/dn-2

[53] Rietveld H M 1969 J. Appl. Cryst. 265

[54] Zlokazov V B and Chernyshev V V 1992 J. Appl. Cryst. 25 447

[55] Domin K, Chan K Y, Yung H, Gubbins K E, Jarek M, Sterczynska A et al 2016 J. Chem. Eng. Data 614252

[56] Suzuki Y, Duran H, Steinhart M, Kappl M, Butt H J and Floudas G 2015 Nano Lett. 151987 